\title{
Review on the Performance of Different Types of Filter in the Presence of Various Noises
}

\author{
Orvila Sarker ${ }^{1}$, Sheuly Akter ${ }^{2}$, Afrina Akter Mishu
}

1,2 Department of Information and Communication Technology, Comilla University, BANGLADESH

${ }^{3}$ Department of Statistics, Comilla University, BANGLADESH

Corresponding Contact:

Email: orvila.sarker@gmail.com

\begin{abstract}
An efficient filter must have the quality of removing noise at the same time preserving the information. On the other hand, noise contaminates in the field of signal processing and communication due to various unwanted nonlinear effects. So study of the characteristics of different types of noise is necessary. Also, how various types of filters response to these noises are also important? In this paper, we have studied the characteristics of four types of noise namely Salt $\&$ pepper noise, Gaussian noise, Speckle noise and Poisson noise and analyzed the performance of four basic types of filters such as Mean filter, Median filter, Wiener filter and Gaussian filter to suppress the noises mentioned previously.
\end{abstract}

Key words

Salt and Pepper noise, Gaussian noise, Speckle noise, Poisson noise, Mean filter, Median filter, Wiener filter, Gaussian filter

$12 / 12 / 2016$

Source of Support: None, No Conflict of Interest: Declared

This article is is licensed under a Creative Commons Attribution-NonCommercial 4.0 International License.

Attribution-NonCommercial (CC BY-NC) license lets others remix, tweak, and build upon work non-commercially,

and although the new works must also acknowledge $\&$ be non-commercial.

\section{INTRODUCTION}

A meaningful image can provide necessary information in many practical applications for example, satellite and Radar communication, medical applications, researches for human evaluation and development, information about a remote geographic region and space research. Unfortunately, when data is collected by image sensors noise get added due to some unexpected or sometimes unavoidable reasons. So, image denoising techniques are necessary to prevent this type of corruption from digital images. Noise arises because of faulty instruments or inappropriate set up of these instruments, loss of a portion or significant amount of data due to signal attenuation or interference etc. By far many noise removal methods have been developed, each having its own merits and demerits. A good noise removal technique has the property of completely removing noise as well as retaining important information such as resolution, edges etc. Before choosing a filter, prior knowledge about noises is desirable (Kaur and Behal, 2013; Verma and Ali, 2013).

Various types of noise present in an image. In this paper, we discuss the characteristics of four types of noise such as Gaussian noise, Salt \& Pepper noise, Speckle noise and Poisson 
noise. Filters like mean, median, Gaussian and Wiener filter are used to remove the noise from image. We also evaluate the noise removal performances of different types of filters.

\section{Characteristics of Four Basic Types of NoIse}

\section{Salt and Pepper noise}

- The reason behind the introduction of Salt \& Pepper noise is mainly because of conversion error in digital systems, bit errors during transmission etc.

- This type of noise doesn't have dependence on image pixels.

- When noisy pixels takes salt value (gray level - 255), it looks black on the image and when they takes pepper value (gray level -0), it appears white on the image.

- Salt and pepper noise detection and removal is complex for binary images because original and noisy images contain the same value 0 or 1 .

- Removing Salt and pepper noise from a grey-scale image is relatively simple because in this case noisy pixels have difference in grey level value. Fig. 1 shows the algorithm of Salt and Pepper noise reduction by a mean filter.

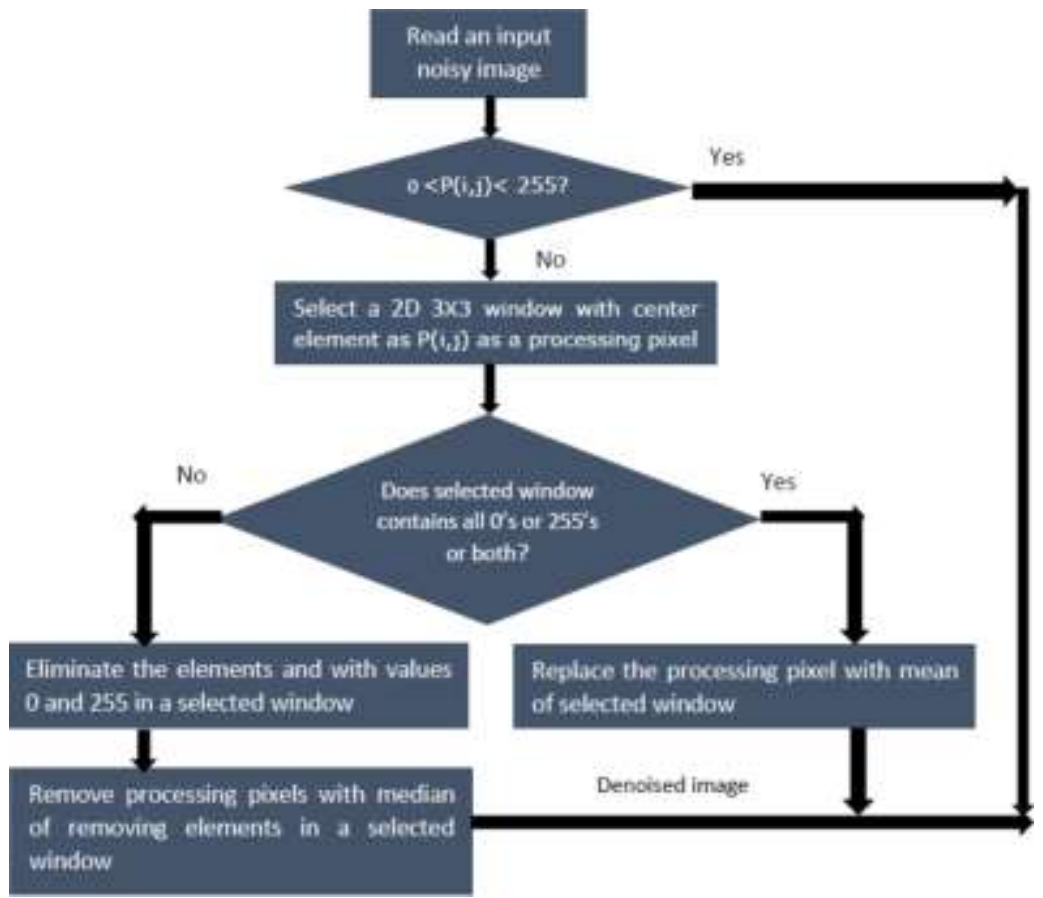

Figure 1: Steps involved in removing Salt and Pepper noise by mean filtering

\begin{tabular}{|l|l|l|l|l|l|l|}
\hline 253 & 208 & 210 \\
\hline 96 & 211 & 34
\end{tabular}$\quad$\begin{tabular}{|l|l|l|}
\hline 253 & 208 & 210 \\
\hline 63 & 105 & 19 \\
\hline
\end{tabular}$\quad$\begin{tabular}{lll}
\hline 63 & 105 & 19 \\
\hline
\end{tabular}

Figure 2: Two $3 \times 3$ image matrices 
Let us consider $3 \times 3$ image matrices which shows in the Fig. 2. Suppose the central value of the matrix 211 is corrupted by Pepper noise. So it will be replaced by value 0 . Consequently, this noise would appear as a dark pixel in the bright region. Fig. 3(a) shows an input image and Fig. 3(b) shows Salt and Pepper noised image.

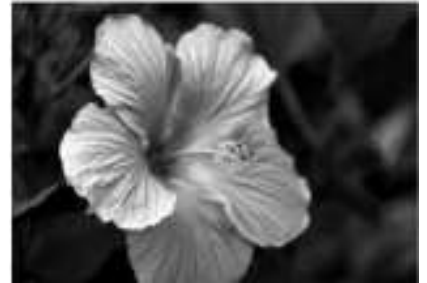

(a)

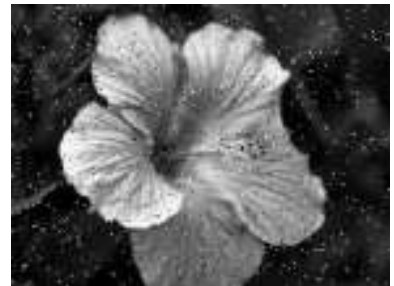

(b)

Figure 3: Salt \& pepper noise (a) original image (b) noisy image

\section{Gaussian noise}

- Random variations in a signal introduces Gaussian noise.

- This type of noise has a bell-shaped probability distribution function. Fig. 4(a) shows an input image and Fig. 4(b) shows Gaussian noised image.

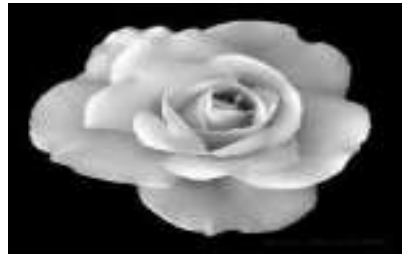

(a)

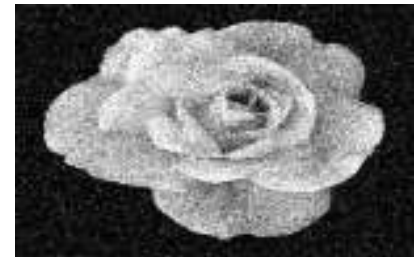

(b)

Figure 4: Gaussian noise (a) original image (b) noisy image

\section{Speckle noise}

- This multiplicative type of noise is observed mostly in coherent imaging systems such as Computer Tomography (CT), acoustics, Magnetic Resonance Imaging (MRI), spectroscopy, laser, digital radiography and more severe in Synthetic Aperture Radar ( SAR) image .

- In case of medical ultrasound imaging, the main reason behind speckle noise is the interference of returning wave collected by the transducer. This occurs mainly due to improper connection between transducer probe and patient body.

- Speckle noise destroys image resolution and edges. Fig. 5(a) shows an input image and Fig. 5(b) shows Speckle noised image.

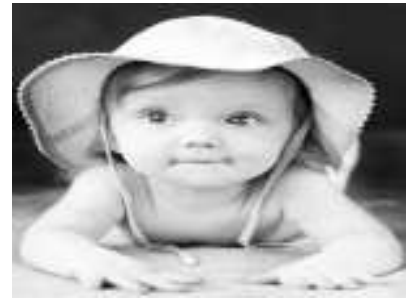

(a)

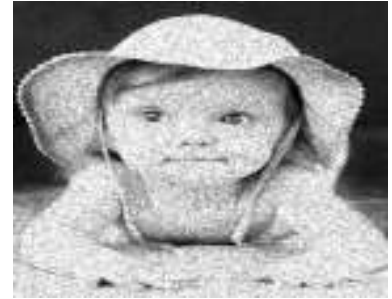

(b)

Figure 5: Speckle noise (a) original image (b) noisy image 


\section{Poisson noise}

- Image sensors count the number of discrete photons incident on it in order to measure image brightness. Poisson noise arises when the arrival of individual photon varies.

- $\quad$ The RMS (Root Mean Square) value of Poisson noise varies according to the SRI (Square Root Intensity) of the image. This kind of noise doesn't depend on other noise sources.

- Analysis of Poisson noise is important in imaging systems that deals with sensor gain. Fig. 6(a) shows an input image and Fig. 6(b) shows Poisson noised image.

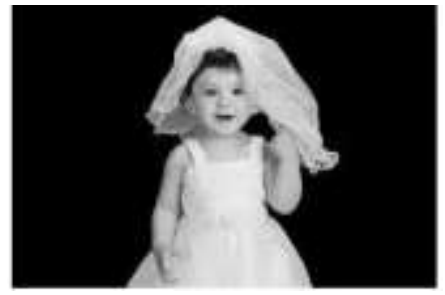

(a)

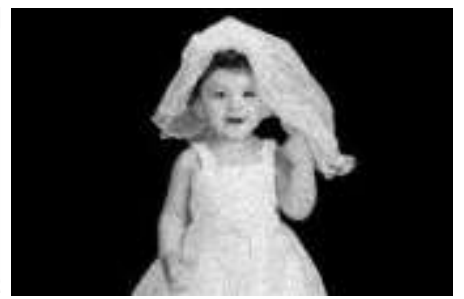

(b)

Figure 6: Poisson noise (a) original image (b) noisy image

\section{Characteristics of Four Basic Types of Filter}

\section{Mean filter}

- A current value of pixel is replaced by the average of all the neighboring pixel values including itself. The same procedure continues for total number of pixels exist in the image.

- Most of the case the window is square shaped.

- Mean filters have the advantage of reducing the intensity variation between adjacent pixels.

- Mean filters are low pass filters. Consequently, they destroy image edges which contain high-frequency components.

- Mean filtering is the least satisfactory method of speckle-noise reduction as it results in loss of detail and resolution.

- The Mean filter is efficient when a portion of the image needs to be analyzed.

\section{Median filter}

- In this filter, a given pixel of the image is replaced with the median value of all neighboring pixel values. At first, all the pixel values is arranged according to numerical order. Then a pixel under consideration is substituted by the middle pixel value.

- It removes noise without reducing the sharp edges of an image if the noise level is less than $50 \%$. A $3 \times 3,5 \times 5$, or $7 \times 7$ kernel of the pixels is used.

- $\quad$ For the same size, median filter causes less blurring as compared to the mean filter.

\begin{tabular}{|l|l|l|l|l|}
\hline 124 & 125 & 127 & 131 & 141 \\
\hline 123 & 125 & 127 & 125 & 136 \\
\hline 116 & 123 & 153 & 126 & 135 \\
\hline 122 & 115 & 123 & 124 & 134 \\
\hline 121 & 126 & 129 & 120 & 119 \\
\hline
\end{tabular}

Figure 7: shows a $5 \times 5$ image matrix. 
Here we obtain an approximate median value of 125 from neighboring pixel values 115123129126121122116123 153. Fig. 8 shows the time domain median filtering of an input signal.

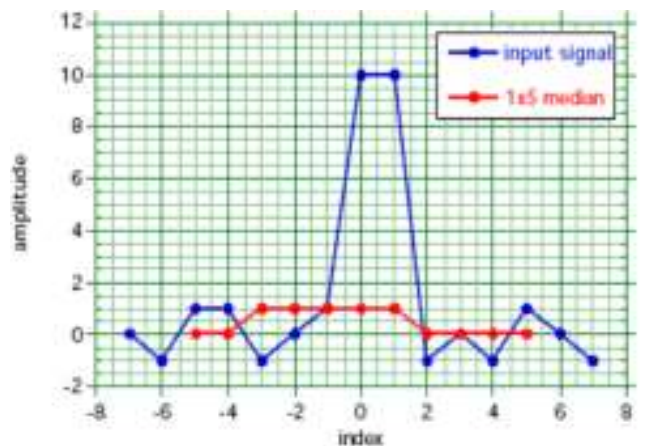

(a)

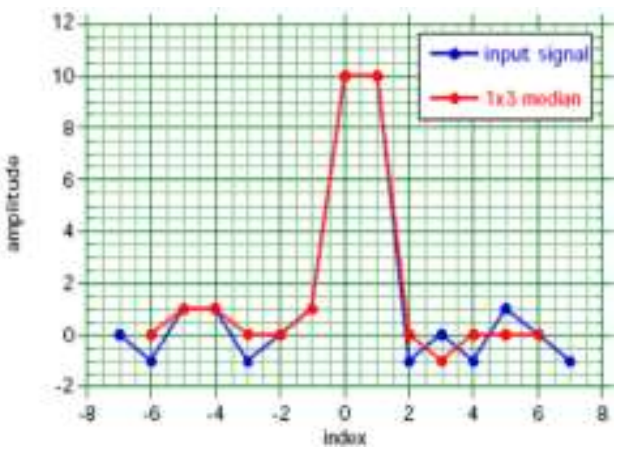

(b)

Figure 8: Mean filtering of an input signal (a) $1 \times 5$ median (b) $1 \times 3$ median

\section{Gaussian filter}

- In a Gaussian filter, the impulse response of the input signal is multiplied by the Gaussian function.

- The Gaussian filter is inappropriate to the applications where the filter bandwidth is much greater than the signal.

- In real-time systems, a delay incurs because incoming samples are needed to fill the filter window before the filter is applied to the signal.

- Gaussian filter is better compared to median filter regarding computational time because multiplying and adding is usually faster than sorting.

\section{Wiener filter}

- Operation within this type of filter requires advance information about noise spectra and the original signal.

- It removes the additive noise while inverts the blurring at the same time.

- Wiener filter performs the deconvolution by inverse filtering and cancels the noise with the compression operation.

\section{RESULTS AND DISCUSSION}

\section{Performance of Mean, Median, Gaussian and Wiener filter}

We took different noise-corrupted input image using MATLAB simulator and analyzed the action of different filters in denoising these noisy images. For the ease of comparison, we include noise contaminated image repeatedly each time we take a different filter. Fig. 9, 11, 13, 15 shows various noise added images and Fig. 10, 12, 14, 16 shows the images filtered by mean, median, Gaussian and Wiener filter respectively. From the overall denoised images, it is observed that speckle noised input image lost a greater amount of resolution when filtered by mean filter compared to others. On the other hand, Gaussian filter performs better in case of Gaussian noised image regarding of resolution and image quality and Salt and pepper noise is best removed by median filter. 


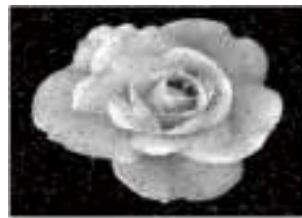

a. Salt \& pepper noise

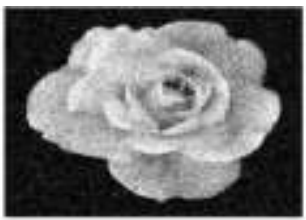

b. Gaussian noise

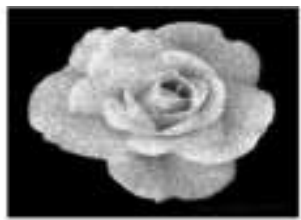

c. Speckle noise

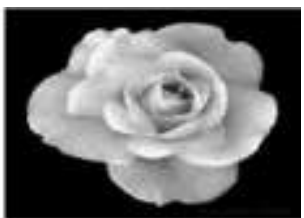

4. Poisson noise

Figure 9: Various noise corrupted input image

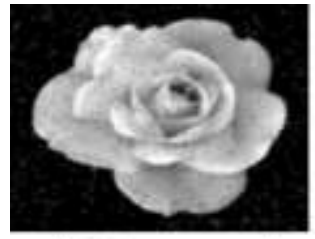

a. Salt \& pepper noise

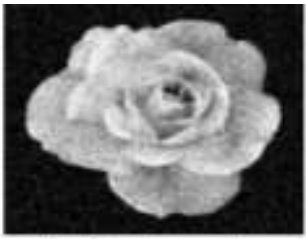

b. Gatussian noise

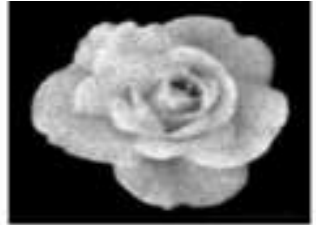

c. Speckle noise

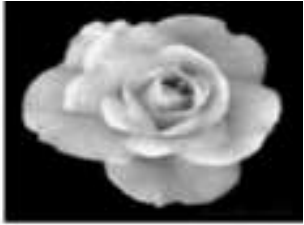

d. Poisson noise

Figure 10: Denoised input image filtered by mean filter

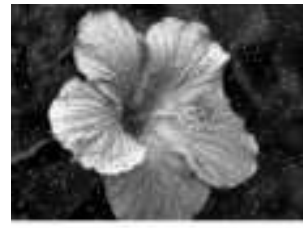

a. Salt \& pepper noise

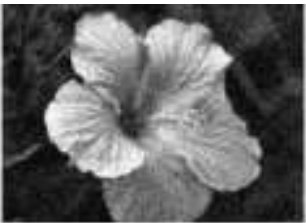

b. Gatısian noise

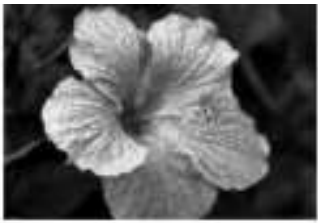

c. Speckle noise

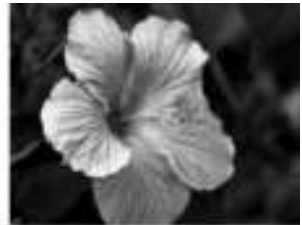

d. Poisson noise

Figure 11: Various noise corrupted input image

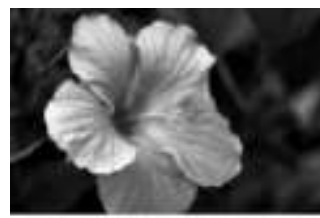

a. Salt \& pepper noise

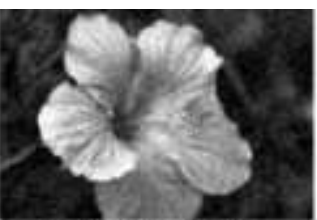

b. Gaussian noise

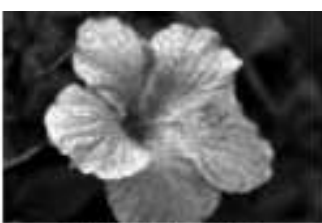

c. Speckle noise

Figure 12: Denoised input image filtered by median filter

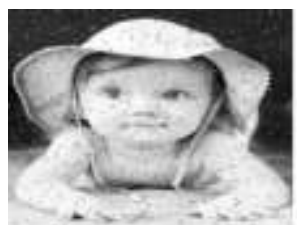

a. Salt \& pepper noise

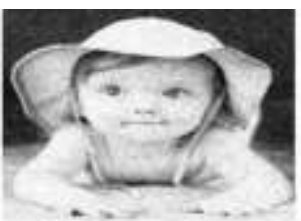

b. Gaussian noise

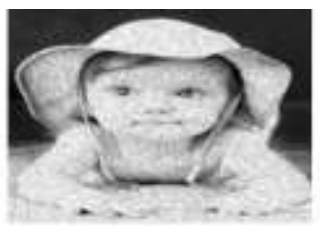

c. Speckle noise

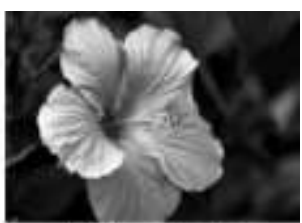

d. Poisson noise

Figure 13: Various noise corrupted input image

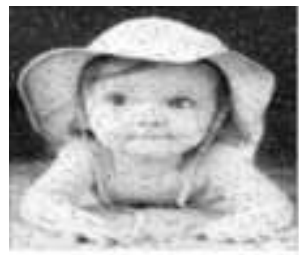

a. Salt \& pepper noise

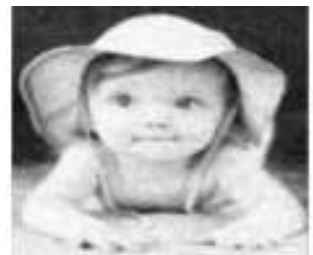

b. Gaussian noise

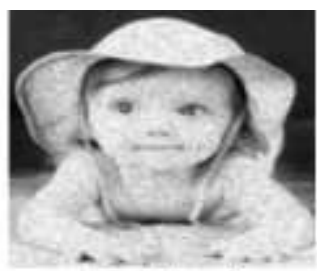

c. Speckle noise

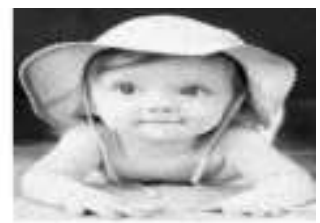

d. Poisson noise

Figure 14: Denoised input image filtered by Gaussian filter

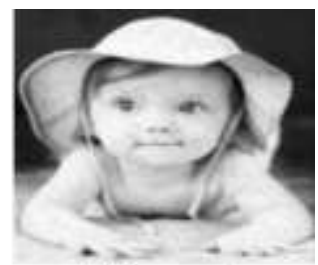

d. Poisson noise 


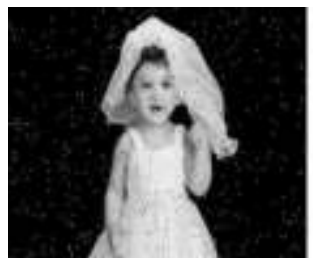

a. Salt \& pepper noise

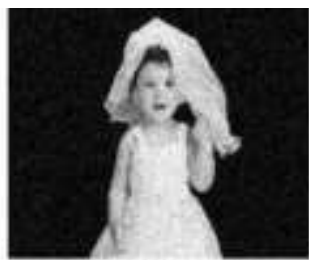

b. Gaussian noise

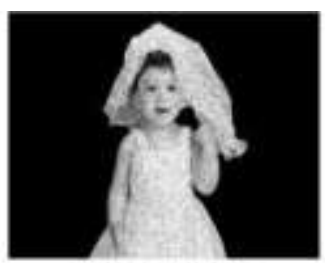

c. Speckle noise

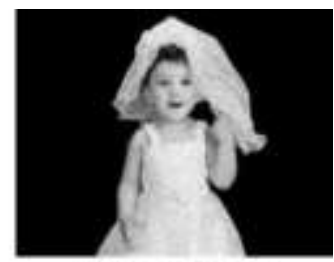

d. Poisson noise

Figure 15: Various noise corrupted input image

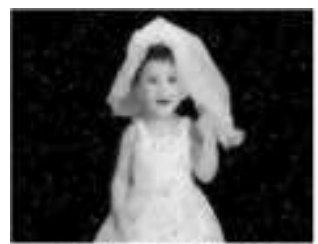

a. Salt \& pepper noise

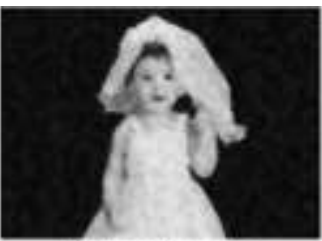

b. Gaussian noise

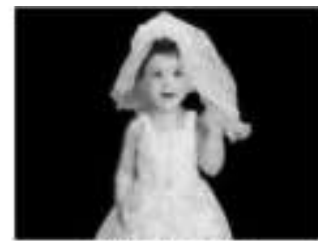

c. Speckle noise

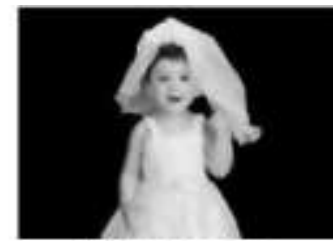

d. Poisson noise

Figure 16: Denoised input image filtered by Wiener filter

\section{CONCLUSION}

In this paper, the characteristics of different types of filter and their filtering action on the application of various noise is discussed. Images carry significant information in each and every field of image processing, so proper noise removing is a crucial step before further processing of image like image restoration, extraction of various features, analysis of edge detection etc. This paper shows the different type of noises that can corrupt the image and different type of filters which are used to recover the noisy image. Different filters show different results after filtering. The mean filter is well suited for Poisson noise. Gaussian filter performs better for Gaussian noise cancellation. Significant Salt and Pepper noise have been filtered by Median filter. Also, Wiener filter works well for removing Poisson and speckle noise. This paper will provide a summarized material for researchers and of course for freshers in the image processing field. Our future work will be to develop and build improved technique which will give better performance than these filters.

\section{References}

A.K. Jain, Fundamentals of Digital Image Processing, Prentice Hall of India, First Edition, 1989.

Ajay Kumar Boyat and Brijendra Kumar Joshi, "A review paper: Noise models in digital image processing,"An International Journal (SIPIJ), Vol 6, No. 2, April 2015.

Alka Pandey "An Overview of Image Denoising and Image Denoising Techniques," Advanced Research in Electrical and Electronic Engineering, Vol. 2, No. 9, April-June 2015.

Hasan S. M. Al-Khaffaf1, Abdullah Z. Talib, Rosalina Abdul Salam, "Removing Salt-and-Pepper Noise from Binary Images of Engineering Drawings," IEEE, 2008.

Klogo Griffith S., Gasonoo Akpeko and Ampomah K. E. Isaac, “On The Performance Of Filters For Reduction Of Speckle Noise In Sar Images Off The Coast Of The Gulf Of Guinea," International Journal of Information Technology, Modeling and Computing $\quad$ (IJITMC), Vol. 1, N. 4, November 2013.

Madhu S. Nair, K. Revathy, and Rao Tatavarti, "Removal of Salt-and Pepper Noise in Images: A New Decision-Based Algorithm,"Proceedings of the International Multi Conference of Engineers and Computer Scientists, Vol I, 19-21 March, 2008.

Manasi Rana, "A review on a statistical analysis of filters on various noises in MRI and USG images (IRJET)," Vol. 02, Issue 03, June 2015. 
Manpreet Kaur and Sunny Behal, "Study of Image Denoising and Its Techniques," IJARCSSE, Vol. 3, Issue 1, January 2013.

Mr. Pawan Patidar, "Image Denoising by Various Filters for Different Noise," International Journal of Computer Applications, Vol. 9, No. 4, November 2010.

Nucharee Premchaiswadi, Sukanya Yimngam, Wichian Premchaiswadi, "A Scheme for Salt and Pepper Noise Reduction on Graylevel and Color Images," Proceedings of the 9th WSEAS International Conference on Signal Processing, Computational Geometry and Artificial Vision.

Rafael C. Gonzalez and Richard E. Woods, Digital Image Processing, $3{ }^{\text {rd }}$ edition, Pearson Publication.

Rohit Verma and Jahid Ali, "A comparative study of various types of image noise and efficient noise removal techniques," International Journal of advanced research in computer science and software engineering, Vol. 3, Issue 10, October 2013.

Ruchika Chandel, Gaurav Gupta, "Image Filtering Algorithms and Techniques: Review," IJARCSSE, Vol. 3, Issue 10, October 2013.

Sarita Danger, “Denoising Techniques: a Comparison," M.S. Thesis, Louisiana State University, 2003.

Sarker, O., \& Jothi, R. (2016). An Improved Image Restoration and Edge Detection Technique. Engineering International, 4(1), 35-40.

Soumya Ruparel, "Image de-noising with 2-d fir filter by using differential evolution algorithm (IJCESR)," Vol. 2, Issue-11, 2015.

Sukhjinder Kaur, "Noise types and various removal techniques," (IJARECE), Vol. 4, Issue 2, February 2015.

ISSN: 2409-3629

Online Archive Link: https://abc.us.org/ojs/index.php/ei/issue/archive 